\title{
Effect of Temperature and Surfactant on the Control Release of Microencapsulated Dye in Lecithin Liposomes. I
}

\author{
A. L. F. Baptista, ${ }^{1}$ P. J. G. Coutinho, ${ }^{2, *}$ M. E. C. D. Real Oliveira, ${ }^{2}$ \\ and J. I. N. Rocha Gomes ${ }^{1}$ \\ ${ }^{1}$ Departamento de Engenharia Têxtil, Universidade do Minho, \\ Guimarães, Portugal \\ ${ }^{2}$ Departamento de Física, Universidade do Minho, Braga, Portugal
}

\begin{abstract}
The objective of our work has been the microencapsulation of dyes with lecithin from soybean, with the formation of liposomes, as a substitute for synthetic auxiliaries so as to improve the quality of the effluent. Current scenarios promote the disintegration and leakage of the liposomes, such as, changes in temperature, $\mathrm{pH}$ and the use of surfactants. Since dyeing process is a mix of all these parameters, we pretended to study each one separately. Rhodamine $6 \mathrm{G}$ fluorescence is known to be concentration quenched through the formation of non-fluorescent dimmers and, additionally, through the energy transfer from rhodamine monomer to these dimmers (Baptista ALF, Coutinho PJG, Real Oliveira MECD, Gomes JINR. Proceedings of 13th International Symposium of Surfactants, SIS 2000, Gainesville, USA, 2000). The temperature, the surfactant and $\mathrm{pH}$
\end{abstract}

*Correspondence: P. J. G. Coutinho, Departamento de Física, Universidade do Minho, Campus de Gualtar, 4710-057 Braga, Portugal; E-mail: pcoutinho@fisica.uminho.pt. 
induce a release of the encapsulated dye resulting in rhodamine dilution and consequently alterations in the dimerization/binding equilibrium. The experimental spectra indicate that rhodamine binds almost completely to liposomes. The decomposition of the rhodamine fluorescence spectra allowed us to determine the percentage of released dye during a simulated dyeing process, and allowed us to conclude that the dimerization process occurs mainly at the inner interfaces. The amount of dye released induced by temperature changes was greater in the presence of surfactant.

\section{INTRODUCTION}

Microencapsulation technology has been widely used in a number of industrial sectors including agriculture, medicine and cosmetics. But the textile industry, although initially slow to exploit the new technology, is now generating innovative ideas and inventions within the field.

In the dyeing of nylon, care must be taken to apply acid dyes uniformly. Uniform dyeing is accomplished by $\mathrm{pH}$ control, slow uniform heating of the dyebath and judicious use of retarding agents.

Liposomes release the microencapsulated dye slowly, promoting a retarding effect, comparable with the one obtained with retarding agents, making them a good alternative to commercial levelling products' (1).

It was since then found that surfactants could influence the behavior of the liposomes increasing the retarding effect on the absorption of the dye by the fibre (2).

It may sound paradoxical but after all the troubles with encapsulation, the release of the dye is also, if not, more problematic. Current scenarios promote the disintegration and leakage of the liposomes, such as, changes in temperature, acidity and the use of surfactants. Since the dyeing process is a mix of all these parameters, in this work we pretended to study each parameter separately.

In our previous work we studied the effect of the surfactant on the vesicles integrity $(3,4)$. We used a combination of fluorescence energy transfer donor and acceptor lipid probes, NBD-PE and Rho-PE and we detected that the size of the liposomes increases when surfactants are added at low concentrations. On the other hand, at higher concentrations of the non-ionic surfactant, $\mathrm{C}_{12} \mathrm{E}_{8}$, we noticed several structural changes in lecithin vesicles, until the formation of mixed micelles.

Encapsulating a xanthene dye in lecithin vesicles produces vesicle solutions that contain dye at very high local concentration, but have a low overall optical density, thereby eliminating reabsorption. Xanthene dyes such as rhodamine $6 \mathrm{G}$ are used as trapped markers inside lecithin vesicles for the purpose of monitoring vesicle integrity. These dyes are useful for this application because they are self-quenched at high local concentrations. When trapped at concentrations of $30 \mathrm{mM}$ inside the aqueous cavity of the vesicle, the fluorescence of rhodamine is nearly $90 \%$ quenched (5). The release of the dye from the vesicle results in dilution of the probe and a large increase in fluorescence intensity.

The mechanism of concentration quenching of these dyes has never been fully elucidated. Understanding the mechanism of concentration quenching may lead to improvements in their application. Many mechanism have been suggested for 
concentration quenching of xanthenes, as collisional quenching, resonance energy transfer, static complex formation, etc., the extent to which these various mechanisms contribute to concentration quenching is difficult to determine due to high complexity of theses systems.

In order to understand the retarding effect of the microencapsulated dye in nylon dyeing, we made several studies on the controlled release of the dye by the liposome namely:

- Study of temperature effect.

- Study of surfactant's effect.

Simultaneously, we also studied the mechanism of formation of the rhodamine dimers inside and outside vesicle membranes responsible for the concentration quenching of this kind of dyes under these conditions.

\section{MATERIALS AND METHODS}

\section{Materials}

Commercial soybean lecithin containing $22 \%$ phosphatidylcholine, $20 \%$ phosphatidylethanolamine, 14\% phosphatidylinositol and 10\% phytoglycolipides was supplied by Stern (USA). The surfactant $\mathrm{C}_{12} \mathrm{E}_{8}$ was purchased from Sigma. Rhodamine 6G was obtained from Merck. Soybean lecithin, rhodamine and the surfactant were used without further purification.

\section{Liposomes Preparation with Rhodamine 6G}

Vesicles were formed by hydrating the lipid film with an aqueous solution (containing a buffer solution $(\mathrm{pH}=5.5)$ and $30 \mathrm{mM}$ of rhodamine $6 \mathrm{G}$ and sonication for $10 \mathrm{~min}$ at $20^{\circ} \mathrm{C}$ and $60 \mathrm{~W}$. All dye, which was not included inside the vesicles, was removed by gel filtration. This preparation resulted in a solution of "microcuvettes" that contained a concentration of dye equal to the concentration of the original solution in which the vesicles were formed. The overall concentration of the rhodamine $6 \mathrm{G}$ in this solution, is calculated using a dilution factor of $3 / 8$ from the gel filtration chromatography and an encapsulation fraction determined from UV-Vis absorption (Shimadzu-UV-3101PC) of 7\%. The size of the liposomes was about $0.8 \mu \mathrm{m}$, measured with a Mastersizer S (Malvern Instruments).

\section{Incorporation of Surfactants into Vesicles}

Zero point zero four millimolar of $\mathrm{C}_{12} \mathrm{E}_{8}$ was incorporated into the liposomes marked with rhodamine $6 \mathrm{G}$ by adding a required volume from a stock surfactant solution to the vesicles. 


\section{Fluorescence Measurements}

In order to study the effect of the temperature on the release of the dye, liposomes marked with rhodamine were submitted to increasing temperature (simulating the dyeing process). Simultaneously, fluorescence intensity was measured with a spectrofluorometer (Spex Fluorolog 212). Rhodamine emission was detected by excitation at $470 \mathrm{~nm}$.

\section{RESULTS AND DISCUSSION}

As seen from Fig. 1, the rhodamine molecules are mostly located in the liposome membranes because its fluorescence spectra are significantly red-shifted when compared to that in water.

When rhodamine is encapsulated in liposomes at a bulk concentration of $10^{-3} \mathrm{M}$ and gel-chromatographed its emission intensity should corresponds to a $2 \times 10^{-5} \mathrm{M}$ bulk rhodamine solution $\left(10^{-3} \mathrm{M} \times 3 / 8 \times 0.07\right)$. But as the observed fluorescence intensity is only 1.5 times greater than that observed for a $1 \times 10^{-6} \mathrm{M}$ bulk solution, there is a self-quenching effect in rhodamine fluorescence emission in liposome membranes.

This rhodamine self-quenching process has been described (6) as originating from non-radiative energy transfer from rhodamine monomers to non-fluorescent rhodamine dimmers.

Considering a binding (6) process of rhodamine in the liposomes and the possibility of dimer formation in both membrane and aqueous phases we will use

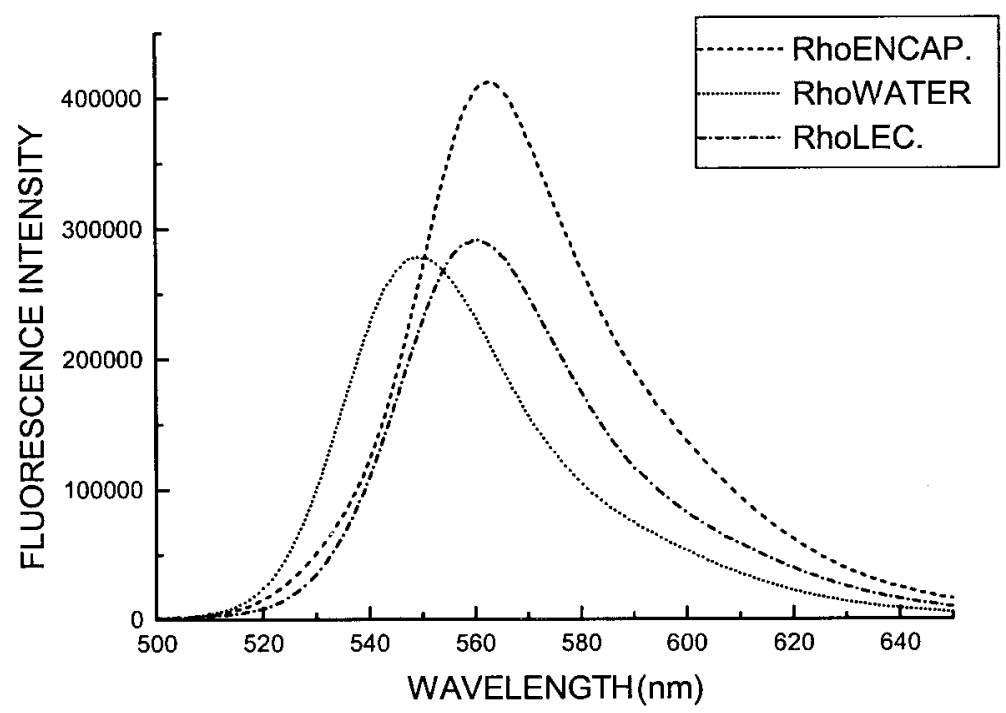

Figure 1. Fluorescence spectra of rhodamine $6 \mathrm{G}$ in different environments. Rhodamine in water $\left(1 \times 10^{-6} \mathrm{M}\right)$, rhodamine encapsulated in soybean lecithin liposomes $\left(2 \times 10^{-5} \mathrm{M}\right)$ and rhodamine in the presence of lecithin $\left(1 \times 10^{-6} \mathrm{M}\right)$. 
the following scheme to describe the rhodamine fluorescence emission behavior in liposomes (Sch. 1), where $k_{q}$ is the dimer quenching rate constant, $K_{\mathrm{RL}}$ is the equilibrium binding constant of rhodamine in soybean liposomes; $K_{D}$ and $K_{\mathrm{DL}}$ are the equilibrium dimerization constants in, respectively, aqueous and lipid media. $k_{\text {isc }}$, $k_{\text {ic }}, k_{F}$ are the usual photophysical non-radiative and radiative rate constants.

Assuming that there is a small fraction of molecules that are excited we can consider that the dimerization/binding equilibrium is not perturbed. Using bulk concentrations we apply this scheme to both interior and exterior liposome membrane interfaces and conclude that, from the mass balance equation:

$$
\left\{\begin{array}{l}
{[R]_{\mathrm{ext}}+\left[R_{\mathrm{L}}\right]_{\mathrm{ext}}+2[D]_{\mathrm{ext}}+2\left[D_{L}\right]_{\mathrm{ext}}=C_{R}^{\mathrm{ext}}=f_{\text {Release }} C_{R}^{0}} \\
{[R]_{\mathrm{int}}+\left[R_{L}\right]_{\mathrm{int}}+2[D]_{\mathrm{int}}+2\left[D_{L}\right]_{\mathrm{int}}=C_{R}^{\text {int }}=\left(1-f_{\text {Release }}\right) C_{R}^{0}}
\end{array}\right.
$$

and using chemical equilibrium:

$$
\left\{\begin{array}{l}
{[R]_{\mathrm{ext}}+K_{\mathrm{RL}} f_{\mathrm{ext}}^{w}[R]_{\mathrm{ext}}[L]_{\mathrm{ext}}+2 K_{D} f_{\mathrm{ext}}^{w}[R]_{\mathrm{ext}}^{2}+2 K_{\mathrm{DL}} f_{\mathrm{ext}}^{m}\left(K_{\mathrm{RL}} f_{\mathrm{ext}}^{w}[R]_{\mathrm{ext}}[L]_{\mathrm{ext}}\right)^{2}=C_{R}^{\mathrm{ext}}} \\
{[R]_{\mathrm{int}}+K_{\mathrm{RL}} f_{\mathrm{int}}^{w}[R]_{\mathrm{int}}[L]_{\mathrm{int}}+2 K_{D} f_{\mathrm{int}}^{w}[R]_{\mathrm{int}}^{2}+2 K_{\mathrm{DL}} f_{\mathrm{int}}^{m}\left(K_{\mathrm{RL}} f_{\mathrm{int}}^{w}[R]_{\mathrm{int}}[L]_{\mathrm{int}}\right)^{2}=C_{R}^{\mathrm{int}}}
\end{array}\right.
$$

where $C_{R}^{0}$ is the initial rhodamine bulk concentration, $f^{w}$ and $f^{m}$ are the conversion factors from bulk concentrations to, respectively, local concentrations in water and at the membrane interface.

The bulk concentrations of lipid can be calculated assuming a small lipid percentage of rhodamine binding $\left(C_{L}=\left[R_{L}\right]+[L] \cong[L]\right)$, and considering that, as the liposome size is large, the number of lipid molecules in the inner and outer

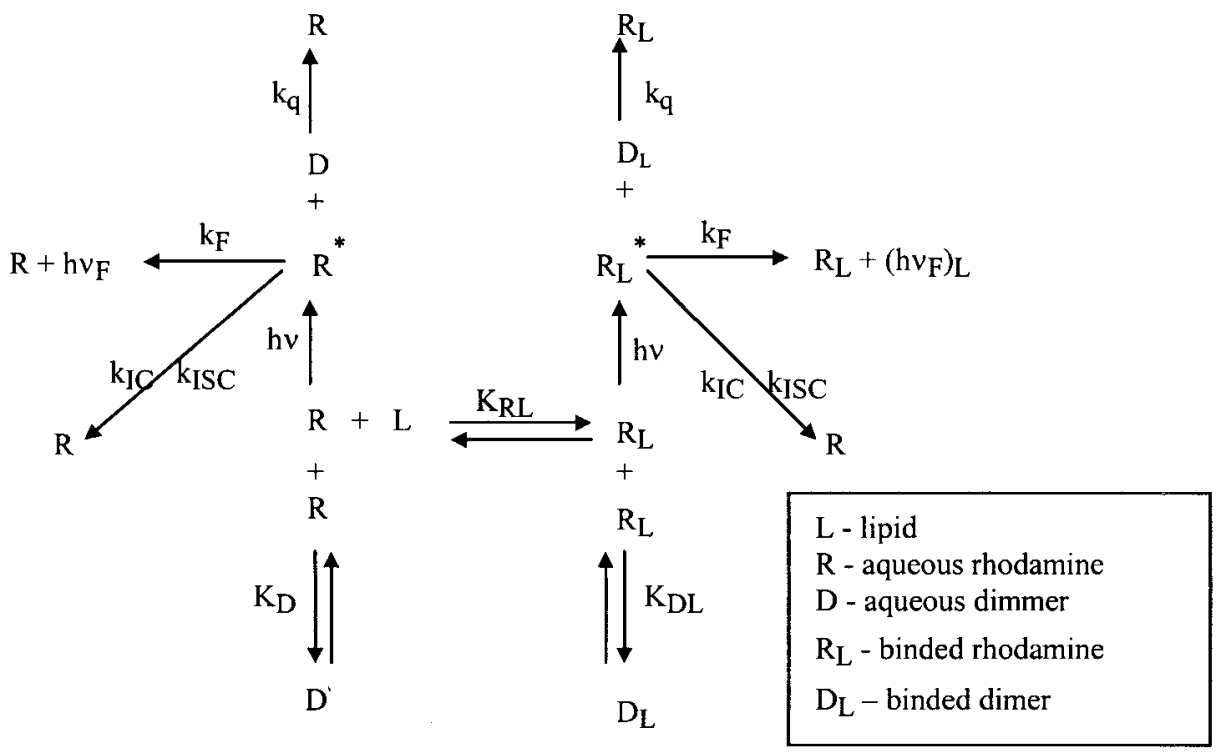

Scheme 1. 
monolayers are equal:

$$
[L]_{\mathrm{ext}}=[L]_{\mathrm{int}}=C_{L} / 2
$$

The concentration conversion factors can be estimated using structural liposome data (Fig. 2):

$$
\begin{aligned}
& f_{\text {int }}^{w}=\frac{V_{\text {solution }}}{V_{\text {int }}^{w}}=\frac{V_{\text {solution }}}{(4 / 3) \pi R_{\text {int }}^{3} N_{\text {liposomes }}}=\frac{V_{\text {solution }}}{(4 / 3) \pi R_{\text {int }}^{3} \frac{C_{L}}{N_{\mathrm{ag}}} V_{\text {solution }} N_{A}}=\frac{N_{\mathrm{ag}}}{(4 / 3) \pi R_{\mathrm{int}}^{3} C_{L} N_{A}} \\
& f_{\text {int }}^{m}=\frac{V_{\text {solution }}}{V_{\text {int }}^{m}}=\frac{N_{\mathrm{ag}}}{(4 / 3) \pi\left(\left(R_{\mathrm{int}}+l_{\mathrm{int}}\right)^{3}-R_{\mathrm{int}}^{3}\right) C_{L} N_{A}} \\
& f_{\mathrm{ext}}^{w}=\frac{V_{\text {solution }}}{V_{\mathrm{ext}}^{w}}=\frac{V_{\text {solution }}}{V_{\text {solution }}-(4 / 3) \pi R_{\mathrm{ext}}^{3} N_{\text {liposomes }}}=\frac{N_{\mathrm{ag}}}{N_{\mathrm{ag}}-(4 / 3) \pi R_{\mathrm{ext}}^{3} C_{L} N_{A}} \\
& f_{\mathrm{ext}}^{m}=\frac{V_{\text {solution }}}{V_{\mathrm{ext}}^{m}}=\frac{N_{a g}}{(4 / 3) \pi\left(R_{\mathrm{ext}}^{3}-\left(R_{\mathrm{ext}}-l_{\mathrm{ext}}\right)^{3}\right) C_{L} N_{A}}
\end{aligned}
$$

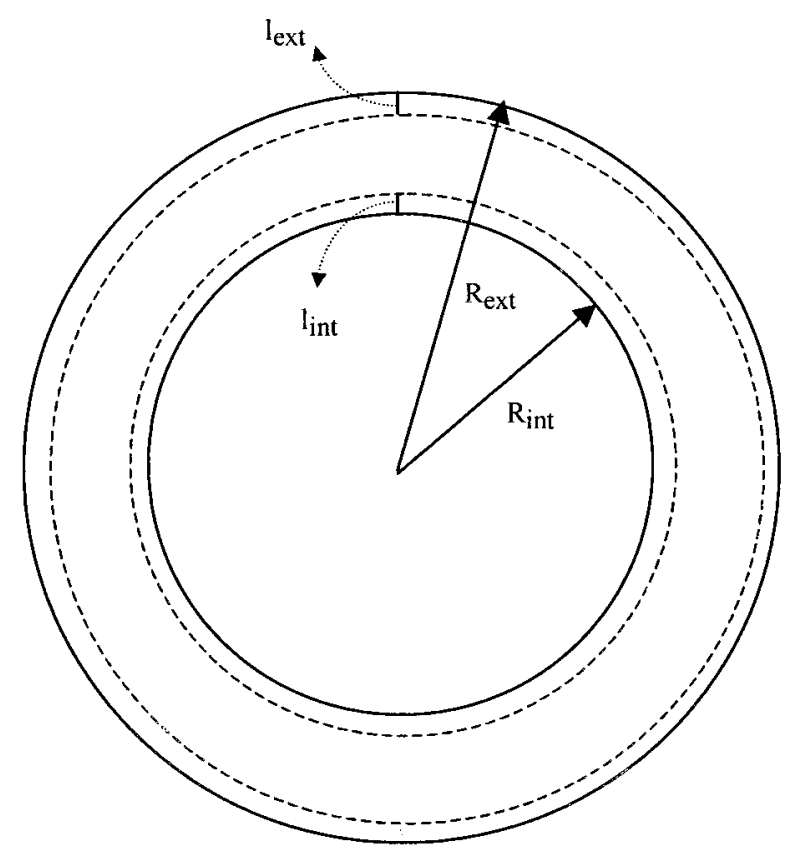

Figure 2. Structural scheme of a liposome: $1_{\mathrm{ext}}$ and $1_{\text {int }}$ are respectively the outer and inner interfaces' thickness and $R_{\text {ext }}$ and $R_{\text {int }}$ are respectively the external and internal radius of the liposome. 
The liposome aggregation number, $N_{\mathrm{ag}}$, can be estimated using an equal internal and external surface area per lipid of $60 \AA^{2}(7)$ :

$$
N_{\mathrm{ag}}=4 \pi \frac{R_{\mathrm{int}}^{2}+R_{\mathrm{ext}}^{2}}{60}
$$

Using for the liposome structural data $l_{\text {ext }}=l_{\text {int }}=10 \AA$ (7), $R_{\text {ext }}=8000 \AA$ (this work), $R_{\text {int }}=(8000-50) \AA$ (7) and $C_{L}=3.75 \times 10^{-4} \mathrm{M}$, we obtain:

$$
f_{\mathrm{int}}^{w}=48 \quad f_{\mathrm{int}}^{m}=1.27 \times 10^{4} \quad f_{\mathrm{ext}}^{w}=1.02 \quad f_{\mathrm{ext}}^{m}=1.26 \times 10^{4}
$$

In a diluted mixture of molecules the total fluorescence emission at wavelength $\lambda$ is given by:

$$
I_{F}(\lambda)=\sum_{i} \varepsilon_{i}\left(\lambda_{\mathrm{exc}}\right) b_{\mathrm{exc}} c_{i} \phi_{F_{i} \alpha_{i}}
$$

where $\varepsilon_{i}$ molar absortivity of species $i, \lambda_{\text {exc }}$ wavelength excitation, $b_{\text {exc }}=1 \mathrm{~cm}$ (optical path), $c_{i}$ concentration of species $i, \phi_{F_{i}}$ fluorescence quantum yield and $\alpha_{i}$ is the fluorescence spectral factor of species $i$.

Thus, the fluorescence emission intensity of rhodamine in liposomes is given by:

$$
\begin{aligned}
I_{F}(\lambda)= & \frac{k_{F}}{k_{F}+k_{\mathrm{IC}}+k_{\mathrm{ISC}}+k_{q} f_{\mathrm{ext}}^{w}[D]_{\mathrm{ext}}}[R]_{\mathrm{ext}} \alpha_{R}^{w}(\lambda) \\
& +\frac{k_{F}}{k_{F}+k_{\mathrm{IC}}+k_{\mathrm{ISC}}+k_{q} f_{\mathrm{int}}^{w}[D]_{\mathrm{int}}}[R]_{\mathrm{int}} \alpha_{R}^{w}(\lambda) \\
& +\frac{k_{F}}{k_{F}+k_{\mathrm{IC}}+k_{\mathrm{ISC}}+k_{q} f_{\mathrm{ext}}^{m}\left[D_{L}\right]_{\mathrm{ext}}}\left[R_{L}\right]_{\mathrm{ext}} \alpha_{\mathrm{RL}}^{\mathrm{ext}}(\lambda) \\
& +\frac{k_{F}}{k_{F}+k_{\mathrm{IC}}+k_{\mathrm{ISC}}+k_{q} f_{\mathrm{int}}^{m}\left[D_{L}\right]_{\mathrm{int}}}\left[R_{L}\right]_{\mathrm{int}} \alpha_{\mathrm{RL}}^{\mathrm{int}}(\lambda)
\end{aligned}
$$

in which the concentration conversion factors were used in order to account for the volume where the quenching process occurs.

Using for diluted rhodamine in water at $20^{\circ} \mathrm{C}$, a natural fluorescence lifetime of $3.7 \mathrm{~ns}$ (8) and a fluorescence quantum yield of 0.72 (9) we estimate that $k_{F}=1.95 \times 10^{8} \mathrm{~s}^{-1}$ and $k_{\mathrm{nr}}=k_{\mathrm{IC}}+k_{\mathrm{ISC}}=7.57 \times 10^{7} \mathrm{~s}^{-1}$. We assume that the variation of $k_{F}$ with temperature is insignificant and for the non-radiactive constant, $k_{\mathrm{nr}}$, we consider an Arrehnius behavior with an activation energy, $E_{a}$, of $23.4 \mathrm{~kJ} \mathrm{~mol}^{-1}$ obtained by Ferreira et al. for rhodamine 3B (10). The dimerization constant of rhodamine in water was determined by Arbeloa et al. (11) as $2.36 \times 10^{3} \mathrm{M}^{-1}$. The corresponding values in the membrane interfaces will be considered as parameters in the fitting of the experimental spectra.

The energy transfer rate constant, $k_{q}$, was obtained by Arbeloa et al. (11) considering also the possibility of rhodamine trimmers formation. Assuming that only dimers are formed we estimate an "effective" $k_{q}$ value in water of $5.5 \times 10^{11} \mathrm{~s}^{-1}$. The viscosity of the liposome interfaces is much higher than water, additionally, there is also, some interaction between rhodamine and the lipids, so the mobility 
of these molecules in liposomes is much lower than in water. As the typical viscosity of membranes is 100 times greater than water, we will use a quenching constant of $5.5 \times 10^{9} \mathrm{~s}^{-1}$.

With temperature, the dimerization equilibrium constant decreases and the quenching rate constant increases (11), thus we neglected this effect because they promote opposing effects on the fluorescence quantum yield.

\section{Temperature Effect}

In Fig. 3A, we observed an increase in fluorescence emission with increasing temperature and a very slight blue shift. This effect is an indication of changes in rhodamine location. The volume of external water is 47 times greater than the internal one, thus, the release of rhodamine from the interior of the liposomes to the external water phase would cause a huge dilution which result in less dimerization and, consequently, in greater fluorescence emission. This is indeed observed (Fig. 3A) but there is no pronounced blue shift of the fluorescence spectra as would be expected according to Fig. 1. The diffusion of rhodamine from the internal to the external liposome interface would not make much difference in the dimerization equilibrium as the volume ratio of these two sub phases is close to one.

We conclude that, either the rhodamine spectral characteristics are very different, namely greater emission in the external than in the internal interface, or the dimerization process is more efficient in the internal interface.

We chose the latter hypothesis and allowed for changes in the spectral factors only in the wavelength position and form while keeping the maximum intensity approximately constant.

In order to fit the experimental spectra we used for rhodamine in water the experimental spectral factors, and those for rhodamine in the internal and external interfaces were fitted to a sum of three gaussian bands with the restriction that the maximum fluorescent intensity could not vary more than $2 \%$ among the three different environments. This procedure assures that the maximum fluorescence intensity of rhodamine does not change too much with the environment avoiding covariation of local concentration and maximum spectral factors. As an approximation we have also considered that the binding process is almost complete and used large value for the binding equilibrium constant. The fitted spectra are on Fig. 3A, as we can see, a good agreement was obtained with the spectral factors represented in Fig. 3B. These are similar to the experimental spectra of rhodamine in the external and internal interfaces. The dimerization equilibrium constants in the external and internal interfaces were respectively, less than $1 \times 10^{-2} \mathrm{M}^{-1}$ and $30.3 \mathrm{M}^{-1}$. This means that in the external interface the dimerization process is not efficient. In fact the liposome structure is a multilamellar one, with an interlayer water thickness of $24 \AA$ (12), confering very different properties to the outer liposome interface because the internal water is much more structured than the external one, which is free. Also the internal concentration conversion factors will be different than the ones previously estimated which affect the value of the fitted dimerization equilibrium constant in the internal interface. 

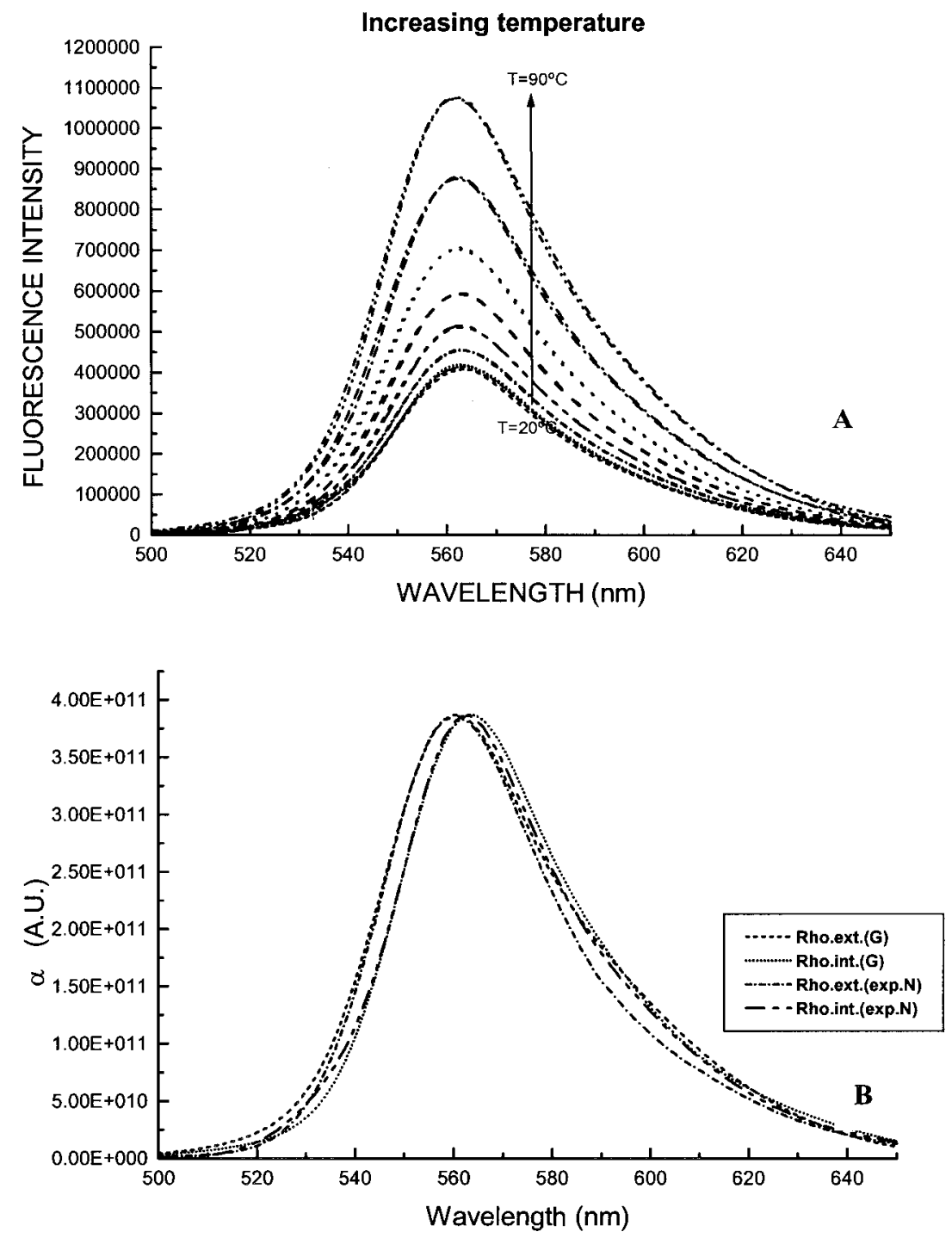

Figure 3. (A) Fitted and experimental fluorescence spectra of rhodamine $6 \mathrm{G}$ encapsulated in soybean lecithin liposomes for each temperature; (B) fitted parameters, where G correspond to gaussian spectral factors and exp. $\mathrm{N}$ the experimental normalized ones.

\section{Surfactant Effect}

In previous work, we found that liposome-surfactant mixtures improve the retarding effect of the dye in the dyeing process of polyamide, liposomes act at the beginning of the process and surfactant at a latter stage, at higher temperatures (4). So we decided to study the release of encapsulated dyes, with temperature, in this kind of mixed structures (3). 


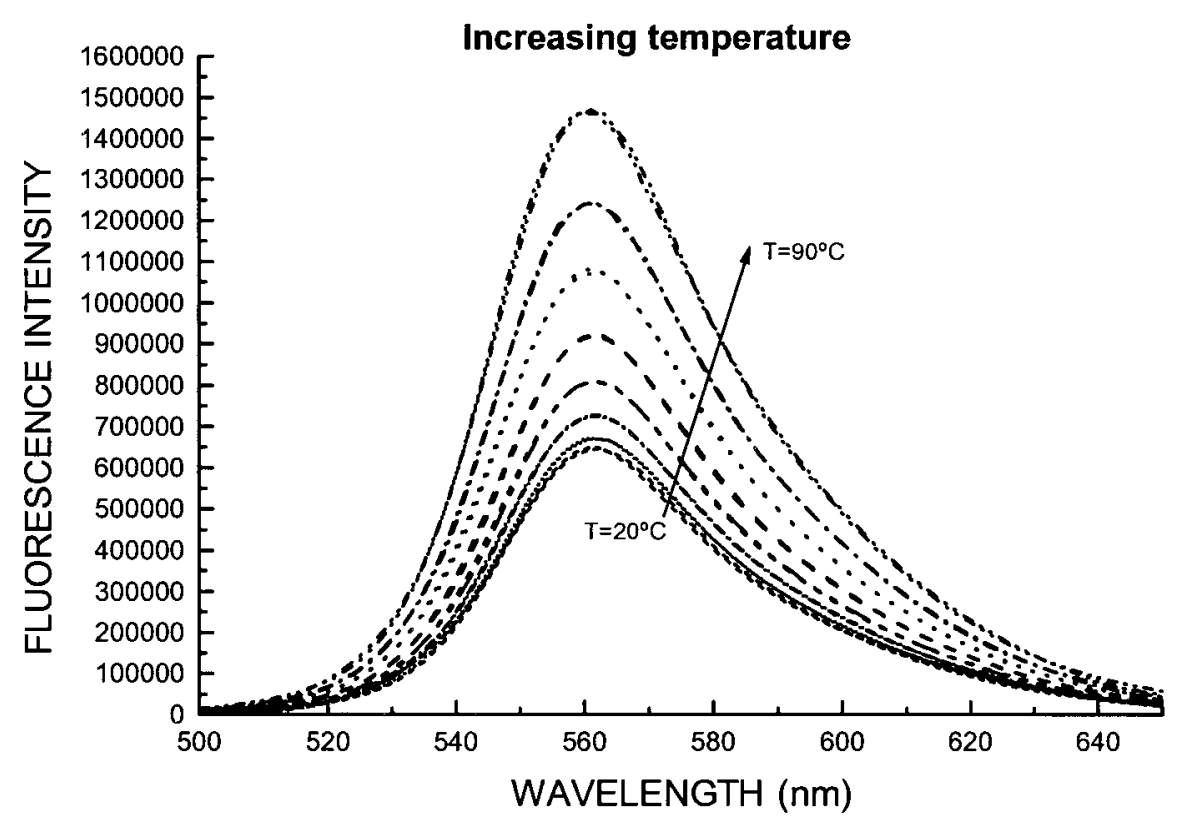

Figure 4. Fitted and experimental fluorescence spectra of rhodamine $6 \mathrm{G}$ encapsulated in mixed soybean lecithin-surfactant liposomes for each temperature.

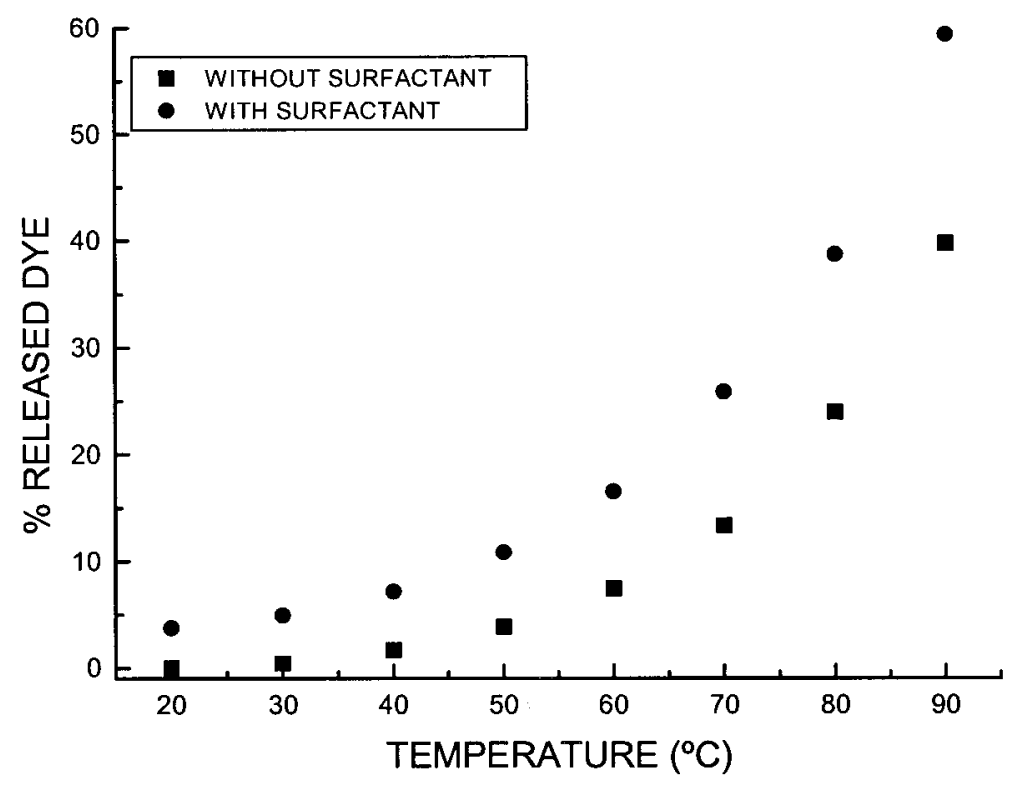

Figure 5. Percentage of released dye from liposomes with and without surfactant. 
The same fitting procedure was used in these liposomes. In Fig. 4, we observed an increase in fluorescence emission with increasing temperature and a very slight blue shift as we have already seen in Fig. 3A.

Comparing the response of the system with and without surfactant (Fig. 5) we can see that the surfactant induces a leakage through the vesicle membrane that increases with temperature.

We have also found that all the spectra are blue-shifted when compared to spectra at corresponding temperatures without surfactant. This can be explained by the non-ionic characteristic of the surfactant.

\section{CONCLUSION}

Temperature and surfactant induce a release of the encapsulated dye resulting in rhodamine dilution and consequently alterations in the dimerization/binding equilibrium.

The amount of dye released induced by temperature changes was greater in the presence of surfactant.

\section{REFERENCES}

1. Gomes JINR, Baptista ALF. Proceedings of the 7th International Conference on Organic Dyes and Pigments, Colorchem' 98, Check Republic, 1998; 11.

2. Gomes JINR, Baptista ALF. Textile Research Journal 2001; 71(2):153-156.

3. Baptista ALF, Coutinho PJG, Real Oliveira MECD, Gomes JINR. Journal of Liposome Research 2000; 10(4):419-429.

4. Baptista ALF, Coutinho PJG, Real Oliveira MECD, Gomes JINR. Proceedings of 13th International Symposium of Surfactants SIS 2000, Gainesville, USA, 2000.

5. Plant AL. Photochemistry and Photobiology 1986; 44(4):453-459.

6. Margalit R, Rotenberg M. Biochimica et Biophysica Acta 1987; 905:173-180.

7. Cornell BA, Midllehurst J, Sparovik F. Biochimica et Biophysica Acta 1980; 598:405.

8. Grawoska E, Tyrzyk J, Bojarski C. Acta Phys Pol A 1980; 57:753.

9. Penzkofer A, Leupacher W. J Lumin 1987; 27:61.

10. Ferreira JAB, Coutinho PJG, Costa SMB, Martinho JMG. Chem Phys 2000; 262:453-465.

11. Arbeloa FL, Ojeda PR, Arbeloa IL. J Chem Soc Faraday Trans 2 1988; 84(12):1903-1912.

12. Rand RP. www.aqueous.labs.brocku.ca/lipid/ 


\section{MARCEl DeKKer, INC. • 270 MAdison AVENUE • New York, NY 10016}

C2003 Marcel Dekker, Inc. All rights reserved. This material may not be used or reproduced in any form without the express written permission of Marcel Dekker, Inc. 